\title{
Bidimensional topology optimization of piezoelectric harvesters
}

\section{Breno V. de Almeida*, Renato Pavanello}

\begin{abstract}
In this article, a piezoelectric energy harvesting device is modeled as a bimorph cantilevered beam, with an intermediate metallic substrate, under plane strain hypothesis. The profile is then optimized by using the Bidirectional Evolutionary Structural Optimization (BESO) method under static loading conditions. Important parameters of the optimization process are varied and the results are displayed and analyzed.
\end{abstract}

\section{Key words:}

piezoeletric, harvester, structural optimization

\section{Introduction}

During the past decade, methods of obtaining energy from the environment have been developed, to transform it into useful electric energy and store it in batteries or capacitors. To achieve these goals, one can increase the energy efficiency of electronic devices, by reusing vibrational waste energy, in other words, harvest it - and the piezoelectric harvester is one of the most interesting vibration-to-energy conversion mechanisms. ${ }^{1}$

A plain strain Finite Element model for a piezoelectric harvesting device was created with a commercial software package - Matlab - and the results were validated with an engineering simulation software ANSYS. ${ }^{2}$ The piezoelectric harvester modeled as a cantilevered beam was then structurally optimized using the $\mathrm{BESO}^{3}$ method for a static excitation of the free end.

\section{Results and Discussion}

A bidimensional bimorph piezoelectric harvester was modeled sing plane strain hypothesis. The model is composed of three layers: one intermediate metallic substrate and two piezoelectric layers with opposite polarities, as shown in image 1.

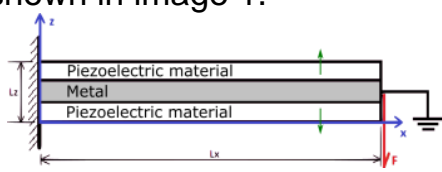

Image 1. Harvester model. The green arrows indicate poling directions and the red one the applied force.

The Finite Element model created in Matlab can successfully return nodal displacements and voltage in 0.8 seconds for a 5000 element of the example in image 1. The results were validated with ANSYS' results. The maximum relative error was $5.33 \cdot 10^{-5}$.

A topology optimization algorithm using the BESO method was then implemented to minimize the inverse conversion factor, as shown below

$$
\begin{array}{ll}
\min & \zeta=1+\frac{\Pi_{S}}{\Pi_{E}} \\
\text { subjected to } & V^{*}-\sum_{i=1} V_{i} x_{i}=0
\end{array}
$$

where $V^{*}$ is the target volume and $x_{i}$ equal to 0.02 for void elements and 1 for solid elements (softkill BESO). ${ }^{3}$ The numerator and denominator of the fraction are the strain energy and electrical energy, respectively.

A $5 \mathrm{~cm}$ long and $1 \mathrm{~cm}$ thick piezoelectric harvester made of PZT-4 and steel ( $1 \mathrm{~cm}$ thick) was optimized for an applied force of $100 \mathrm{~N}$, with a target volume of $55 \%$.

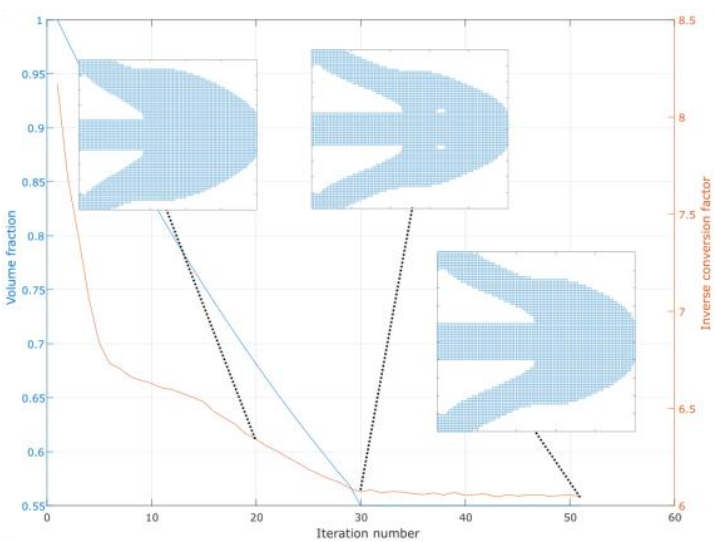

Image 2. Harvester structural optimization convergence and topologies.

The inverse conversion factor had a $24 \%$ decrease with the optimization process after removing $45 \%$ of the total material, as shown in image 2 . The final topology is also shown in the image.

Two structural parameters were varied, to better understand the efficiency of the proposed topology optimization of piezoelectric harvesters - the piezoelectric to metallic stiffness ratio and the thickness to length ratio. An interesting observation is that the smaller the first ratio and the greater the second one, the more significant and smoother becomes the convergence.

\section{Conclusions}

A finite element model for the piezoelectric harvester in plain strain hypothesis was modeled and validated. A piezoelectric harvester was then successfully structurally optimized, improving the energy conversion ratio by $24 \%$. Design parameters of a harvester were varied for a better understanding of the optimization process. The next logical step is to optimize for harmonic base excitation structural optimization, and possibly an optimization for a periodic nonharmonic or even transient excitation could be further studied.

\section{Acknowledgement}

The authors are grateful for the financial support by the Brazilian National Research Council (CNPq) for this work.

${ }^{1}$ Erturk, A and Inman, DJ. Piezoelectric Energy Harvesting. Chichester: John Wiley \& Sons Ltd; 2011.

${ }^{2}$ ANSYS $®$ Mechanical APDL, Realease 16, ANSYS Inc.

${ }^{3}$ Huang X, Xie, YM. Topology Optimization of Continuum Structures: Methods

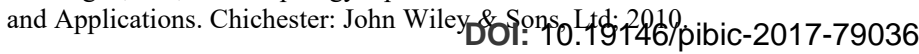

